\title{
The line-of-sight distribution of water in the SgrB2 complex
}

\author{
C. Comito ${ }^{1}$, P. Schilke ${ }^{1}$, M. Gerin ${ }^{2}$, T. G. Phillips ${ }^{3}$, J. Zmuidzinas ${ }^{3}$, and D. C. Lis ${ }^{3}$ \\ 1 Max-Planck-Institut für Radioastronomie, Auf dem Hügel 69, 53121 Bonn, Germany \\ 2 Laboratoire de Radioastronomie Millimétrique, Observatoire de Paris and École Normale Supérieure, 24 rue Lhomond, \\ 75231 Paris Cedex 05, France \\ ${ }^{3}$ California Institute of Technology, Downs Laboratory of Physics 320-47, Pasadena, CA 91125, USA
}

Received 17 December 2002 / Accepted 27 February 2003

\begin{abstract}
We report the detection, with the Caltech Submillimeter Observatory ${ }^{\star}$, of the $894-\mathrm{GHz} \mathrm{HDO}\left(1_{1,1}-0_{0,0}\right)$ transition, observed in absorption against the background continuum emission of the SgrB2 cores M and N. Radiative transfer modeling of this feature, together with the published data set of $\mathrm{mm}$ and submm $\mathrm{HDO}$ and $\mathrm{H}_{2}^{18} \mathrm{O}$ transitions, suggests that groundstate absorption features from deuterated and non-deuterated water trace different gas components along the line of sight. In particular, while the HDO line seems to be produced by the large column densities of gas located in the SgrB2 warm envelope, the $\mathrm{H}_{2}^{18} \mathrm{O}$ ground-state transition detected by SWAS and KAO at $548 \mathrm{GHz}$ (Neufeld et al. 2000; Zmuidzinas et al. 1995a) is instead a product of the hot, diffuse, thin gas layer lying in the foreground of the SgrB2 complex.
\end{abstract}

Key words. astrochemistry - ISM: individual objects: SgrB2 - ISM: abundances - ISM: molecules

\section{Introduction}

The SgrB2 cloud is one of the most massive star forming regions in our Galaxy. It has many unique characteristics, among them an exceptional chemistry. Several species $(\mathrm{FeO}$, Walmsley et al. 2002; $\mathrm{NH}_{2}$, van Dishoeck et al. 1993; HF, Neufeld et al. 1997; NH, Goicoechea et al. 2000) have, in spite of searches elsewhere, been detected only toward this source. One possible explanation for this enigmatic chemistry is the existence of a foreground layer of hot gas, which has been observed e.g. in high-excitation ammonia transitions (cf. Hüttemeister et al. 1995). However, since this layer has the same velocity as the ambient gas in SgrB2, it has been proven difficult to assess its importance for the chemistry of many species. In particular its importance for the water chemistry has been a matter of recent debate (Ceccarelli et al. 2002, hereafter $\mathrm{C} 02$; Neufeld et al., in prep.). While its temperature and density are well constrained by observational data (cf. Hüttemeister et al. 1995), its column density and spatial extent have remained elusive. In this paper we are able to determine its importance for water chemistry and provide constraints on its column density and spatial width, by modeling the HDO and $\mathrm{H}_{2}^{18} \mathrm{O}$ emission and absorption.

Water is known to be a fundamental ingredient of the interstellar medium. It is a major coolant of star-forming clouds

Send offprint requests to: C. Comito, e-mail: ccomito@mpifr-bonn.mpg.de

* The CSO is operated by the California Institute of Technology under funding from the National Science Foundation, Grant No. AST-9980846.
(Ceccarelli et al. 1996) and therefore it affects the dynamical evolution of the clouds. A ubiquitous tracer of shock-heated gas, it dramatically influences the chemistry in shocked regions (cf. Neufeld \& Melnick 1987; Bergin et al. 1998). Although direct, ground-based observations of non-masing water lines are made extremely difficult by atmospheric absorption, $\mathrm{H}_{2} \mathrm{O}$ abundances can be estimated via observations of isotopomers, such as $\mathrm{H}_{2}^{18} \mathrm{O}$ (e.g. Phillips et al. 1978; Jacq et al. 1988 and 1990; Gensheimer et al. 1996), and, in recent years, by the availibility of satellites such as ISO (e.g. van Dishoeck \& Helmich 1996; Cernicharo et al. 1997; Wright et al. 2000) and notably SWAS (cf. Melnick et al. 2000; Snell et al. 2000a, 2000b; Neufeld et al. 2000, hereafter N00), but also by airborne observations of $\mathrm{H}_{2}^{18} \mathrm{O}$ (e.g. Zmuidzinas et al. 1995a, hereafter Z95a; Timmermann et al. 1996). However, the deuterated counterpart of water, HDO, presents many features observable from ground in the $\mathrm{cm}, \mathrm{mm}$ and submm wavelength atmospheric windows (cf. Henkel et al. 1987; Jacq et al. 1990; Schulz et al. 1991; Helmich et al. 1996; Jacq et al. 1999; Pardo et al. 2001), and can also be used as a tracer of water abundance under the assumption that both the deuterated and nondeuterated species are spatially coexistent, and that their abundance ratio is constant throughout the region of interest.

We report here the detection, with the Caltech Submillimeter Observatory, of the ground-state $\left(1_{1,1}-0_{0,0}\right)$ transition of deuterated water, observed in absorption against the background continuum sources $\operatorname{SgrB} 2(\mathrm{M})$ and $\operatorname{SgrB} 2(\mathrm{~N})$. The radial velocity of the observed HDO feature suggests their direct connection to the SgrB2 complex. 
In Sect. 3.1 we estimate the column density of the absorbing HDO using classical absorption line assumptions, based on the $894-\mathrm{GHz}$ feature. Moreover, thanks to the $\mathrm{H}_{2}^{18} \mathrm{O}$ SWAS data of $\mathrm{NO0}$, we are able to estimate the $[\mathrm{HDO}] /\left[\mathrm{H}_{2} \mathrm{O}\right]$ ratio towards the SgrB2 complex (Sect. 3.2). The interpretation of these results, however, depends critically on the assumptions on the location of the absorbing gas. In fact, the observed absorption could be produced both in the above-mentioned hot gas layer, and in the warm envelope of molecular gas in which the two main cores are known to be embedded. Moreover, the assumption that deuterated and non-deuterated water be spatially coexistent is probably incorrect in the SgrB2 cloud. In Sect. 4, we make use of our CSO 894-GHz data, as well as of the published $\mathrm{HDO}$ and $\mathrm{H}_{2}^{18} \mathrm{O}$ observations carried out at $\mathrm{mm}$ and submm wavelengths, to model the distribution of water throughout the whole SgrB2 complex in its three component: hot cores, warm envelope and hot layer, using a state-of-the-art radiative transfer model. The results are discussed in Sect. 5 .

\section{Observations}

The observations were carried out at the Caltech Submillimeter Observatory atop Mauna Kea, Hawaii, on July 28, 2001. Scans were taken, using the chopping secondary with a throw of $4^{\prime}$, towards the coordinates $\alpha_{\mathrm{J} 2000}=17^{\mathrm{h}} 47^{\mathrm{m}} 20.206, \delta_{\mathrm{J} 2000}=$ $-28^{\circ} 23^{\prime} 05^{\prime \prime} 27$ for SgrB2(M), and $\alpha_{\mathrm{J} 2000}=17^{\mathrm{h}} 47^{\mathrm{m}} 20^{\mathrm{s}} 389$, $\delta_{\mathrm{J} 2000}=-28^{\circ} 22^{\prime} 22^{\prime \prime} 25$ for SgrB2$(\mathrm{N})$. At the observing frequency of $893.6 \mathrm{GHz}$, the CSO 10.4-m antenna has a HPBW of about $10^{\prime \prime}$.

The 500-MHz facility AOS was used as backend, providing a velocity resolution of $\sim 0.5 \mathrm{~km} \mathrm{~s}^{-1}$. System temperatures varied between 7500 and $8000 \mathrm{~K}$. Several different LO settings were needed in order to cover a wide enough velocity range, and to access at least two, namely the $65-$ and $81-\mathrm{km} \mathrm{s}^{-1}$, of the several velocity components known to exist in the gas clouds lying on the line of sight in the direction of the two cores (e.g., Whiteoak \& Gardner 1979; Martín-Pintado et al. 1990; Greaves et al. 1992; Tieftrunk et al. 1994). Also, the use of various LO settings allowed us to rule out possible contamination from signal coming from the image sideband. In total, the range $-60 \mathrm{~km} \mathrm{~s}^{-1} \lesssim v_{\mathrm{LSR}} \lesssim 140 \mathrm{~km} \mathrm{~s}^{-1}$ was covered. However, the low signal-to-noise ratio does not allow an analysis of the other velocity components, e.g. at $\sim 0$ and $\sim 100 \mathrm{~km} \mathrm{~s}^{-1}$, towards which water absorption has been detected (cf. N00).

We would also like to mention that the second ground-state rotational transition of HDO, the $1_{0,1}-0_{0,0}$ at $465 \mathrm{GHz}$, has been searched for by us as well as by other authors (e.g., E. Bergin and collaborators, priv. comm.). A clear detection could not be achieved, however all data sets are consistent in indicating that the $465-\mathrm{GHz}$ transition shows up as a weak emission line.

Pointing is a delicate issue for the $850-\mathrm{GHz}$ receiver (Kooi et al. 2000) at the CSO, therefore it was checked rather often through 5-point maps of Mars, located only $\sim 11^{\circ}$ away from our target sources. The accuracy of our pointing is confirmed by the continuum levels we measure towards the two cores ( $21 \mathrm{~K}$ for $\mathrm{M}$ and $13 \mathrm{~K}$ for $\mathrm{N}$, in units of main-beam temperature), that match, within $20 \%$ error, with the values inferred by a map of the $350-\mu \mathrm{m}$ continuum emission acquired with
SHARC $^{1}$ at the CSO (Dowell et al. 1999). Observing Mars also allowed us to measure the beam efficiency of the telescope, which we found to be around $30 \%$.

\section{Results}

\subsection{Determination of HDO column densities}

Figure 1 displays two spectra of the ground-state $1_{1,1}-0_{0,0}$ transition of HDO, observed in absorption against the continuum emission of SgrB2(N) (upper panel) and SgrB2(M) (lower panel).

The determination of column densities from absorption lines is in general more accurate, with respect to the quantities inferred from the analysis of emission lines, because the calculated value does not depend on the excitation temperature of the line, $T_{\mathrm{ex}}$, as long as this is negligible with respect to the temperature of the background continuum source, $T_{\mathrm{C}}$ : in other words, no assumptions need be made about the physical state of the gas, except that $T_{\mathrm{ex}} \ll T_{\mathrm{C}}$ and that only the ground state of the molecule is populated (we will show in Sect. 4 that neither holds for a large part of the warm envelope). The total column density of the absorbing species is given by:

$N_{\mathrm{tot}}=\frac{8 \pi v^{3}}{A_{\mathrm{ul}} c^{3}} \Delta v \frac{g_{1}}{g_{\mathrm{u}}} \tau$.

The opacity of an absorption line can be determined by:

$\tau=-\ln \left[1-\frac{T_{\mathrm{L}}}{T_{\mathrm{C}}}\right]$

where $T_{\mathrm{L}} / T_{\mathrm{C}}$ is the line-to-continuum ratio. Therefore, Eq. (1) only depends on $T_{\mathrm{L}} / T_{\mathrm{C}}$ and on the line width, $\Delta v$, quantities that can be easily derived from a Gaussian fit of the optical depth. Note that, at this stage, no assumptions have been made about the actual location (warm envelope or hot layer) of the gas producing the observed features.

The fit to the $65-$ and $81-\mathrm{km} \mathrm{s}^{-1}$ components of $\mathrm{N}$, and to the $65-\mathrm{km} \mathrm{s}^{-1}$ component of $\mathrm{M}$ are shown in Fig. 1, while the derived parameters are summarized in Table 1. The relative values of the total column density are listed in Table 2 . Studies of the 1.3-mm dust emission in the region (IRAM 30-m maps, Gordon et al. 1993) indicate that the column density of $\mathrm{H}_{2}, N\left(\mathrm{H}_{2}\right)$, reaches values of $2.2 \times 10^{24} \mathrm{~cm}^{-2}$ towards SgrB2(M), and $7.5 \times 10^{24} \mathrm{~cm}^{-2}$ towards $\operatorname{SgrB} 2(\mathrm{~N})$. Such results agree, within a factor of 2 , with previous estimates obtained by Goldsmith et al. (1987) based on lower-spatial-resolution $1.3-\mathrm{mm}$ continuum maps. The relative abundance of gas-phase HDO displayed in the last column of Table 2 has been estimated on the basis of the above mentioned values of $N\left(\mathrm{H}_{2}\right)$. The estimate takes into account that the amount of gas we "see" through the HDO absorption is only a half of the actual amount of gas measured through the core. We take the error on the HDO abundance to be of $\sim 50 \%$.

\footnotetext{
1 Submillimeter High Angular Resolution Camera.
} 


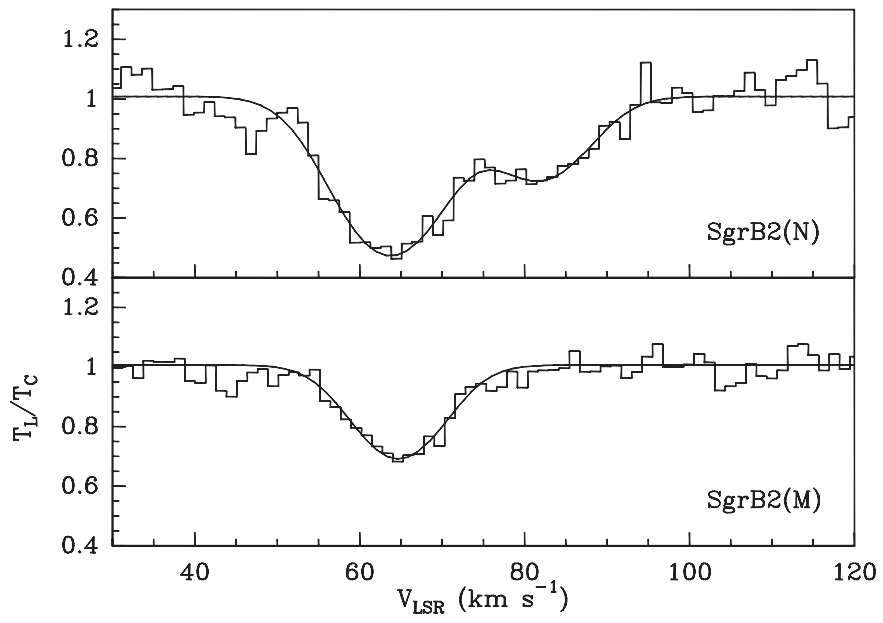

Fig. 1. The $894-\mathrm{GHz}$ HDO $\left(1_{1,1}-0_{0,0}\right)$ transition, observed in absorption against the continuum emission of SgrB2(M) (lower panel) and of SgrB2(N) (upper panel). The $y$-scale gives the line-to-continuum $\left(T_{\mathrm{L}} / T_{\mathrm{C}}\right)$ ratio. The absorption towards $\mathrm{SgrB} 2(\mathrm{M})$ shows one velocity component only, around $65 \mathrm{~km} \mathrm{~s}^{-1}$, while the gas in front of $\operatorname{SgrB} 2(\mathrm{~N})$ shows an additional component around $81 \mathrm{~km} \mathrm{~s}^{-1}$. Such behaviour has been found for other molecular species as well (e.g. $\mathrm{H}_{2} \mathrm{CO}$, see Martín-Pintado et al. 1990). The Gaussian fits to the optical depth of the lines are shown as solid curves, and the parameters derived from the fit are summarized in Table 1.

Table 1. Results of the fits to the optical depth of the HDO absorption features shown in Fig. 1. Columns 2 to 4 give, respectively, the radial velocity of the absorbing gas, the line opacity and the line width. The fits have been performed with the method ABSORPTION from the GILDAS CLASS package.

\begin{tabular}{cccc}
\hline \hline SOURCE & $\begin{array}{c}v_{\text {LSR }} \\
\left(\mathrm{km} \mathrm{s}^{-1}\right)\end{array}$ & $\tau$ & $\begin{array}{c}\Delta v \\
\left(\mathrm{~km} \mathrm{~s}^{-1}\right)\end{array}$ \\
\hline SgrB2(M) & $64.7 \pm 0.5$ & $0.38 \pm 0.04$ & $12.5 \pm 1.3$ \\
SgrB2(N) & $63.7 \pm 0.5$ & $0.77 \pm 0.05$ & $14.6 \pm 1.3$ \\
SgrB2(N) & $82.1 \pm 0.9$ & $0.32 \pm 0.04$ & $13.0 \pm 1.7$ \\
\hline
\end{tabular}

\subsection{The $[\mathrm{HDO}] /\left[\mathrm{H}_{2} \mathrm{O}\right]$ ratio}

N00 have observed, with the Submillimeter Wave Astronomy Satellite (SWAS), the ground-state $1_{1,0}-1_{0,1}$ transition of $\mathrm{o}-\mathrm{H}_{2}^{18} \mathrm{O}(547.7 \mathrm{GHz})$, in absorption against the continuum emission of SgrB2. They were therefore able to estimate the column density of $\mathrm{H}_{2}^{18} \mathrm{O}$ over a wide range of radial velocities (from -120 to $20 \mathrm{~km} \mathrm{~s}^{-1}$ ).

We use the $\mathrm{H}_{2}^{18} \mathrm{O}$ spectrum of $\mathrm{N} 00$ to estimate the $[\mathrm{HDO}] /\left[\mathrm{H}_{2} \mathrm{O}\right]$ ratio in the gas components with radial velocities of 63 and $81 \mathrm{~km} \mathrm{~s}^{-1}$. The comparison between the two data sets is not straightforward, since the spatial resolution of SWAS, 3.3 $\times 4.5$, is such that the emission from SgrB2(M) and SgrB2(N), which are $\sim 47^{\prime \prime}$ apart, is not resolved. To get around this problem, we simply sum up the HDO scans towards M and $\mathrm{N}$ weighted by the attenuation of the circularized 3.9 SWAS beam, and compare the resulting spectrum (hereafter $\mathrm{M}+\mathrm{N}$ ) to that of N00. Such procedure is based on the assumption that the absorbing cloud is extended and uniform over the SWAS beam size. This is a reasonable approximation for the $65-\mathrm{km} \mathrm{s}^{-1} \mathrm{com}-$ ponent, which is known to be extended over both SgrB2(M) and $\mathrm{N}$. However, the $81-\mathrm{km} \mathrm{s}^{-1}$ gas component is definitely smaller than the SWAS beam (it is only observed towards the northern core).

Table 2. Column densities of HDO calculated from the absorption features shown in Fig. 1. Column 2 gives the radial velocity of the absorbing gas component. The values of the column density (Col. 3) are derived, using Eq. (1), assuming that only the ground-state level of HDO is populated. Column 4 lists the estimated HDO abundance, calculated assuming a $\mathrm{H}_{2}$ column density of $2.2 \times 10^{24} \mathrm{~cm}^{-2}$ towards SgrB2(M) and $7.5 \times 10^{24} \mathrm{~cm}^{-2}$ towards SgrB2(N) (Gordon et al. 1993). The estimated error of the HDO abundance is of order $50 \%$.

\begin{tabular}{cccc}
\hline \hline SOURCE & $\begin{array}{c}v_{\text {LSR }} \\
\left(\mathrm{km} \mathrm{s}^{-1}\right)\end{array}$ & $\begin{array}{c}N(\mathrm{HDO}) \\
\left(\times 10^{13} \mathrm{~cm}^{-2}\right)\end{array}$ & $\begin{array}{c}{[\mathrm{HDO}]} \\
\left(\times 10^{-11}\right)\end{array}$ \\
\hline SgrB2(M) & 64.7 & $1.2 \pm 0.2$ & 1.1 \\
SgrB2(N) & 63.7 & $2.8 \pm 0.3$ & 0.7 \\
SgrB2(N) & 82.1 & $1.1 \pm 0.2$ & 0.3 \\
\hline
\end{tabular}

Figure 2 compares the HDO absorption features towards $\mathrm{M}+\mathrm{N}$ (upper panel) with the $548-\mathrm{GHz}$ o- $\mathrm{H}_{2}^{18} \mathrm{O}$ ground-state line observed by N00 (lower panel). The solid curves in Fig. 2 represent the two-component Gaussian fits to the opacity of the observed features, and the fitting parameters are summarized in Table 3. Again, we used Eq. (1) to calculate the total column density of the two species, assuming that, for both of them, only the ground-state level is populated. The results are summarized in Table 4 . The last column of Table 4 shows the estimated value of the $[\mathrm{HDO}] /\left[\mathrm{H}_{2} \mathrm{O}\right]$ ratio for the two velocity components of the gas, calculated assuming that $\left[{ }^{16} \mathrm{O}\right] /\left[{ }^{18} \mathrm{O}\right]=$ $261 \pm 20$ (Whiteoak \& Gardner 1981), and that $\left[0-\mathrm{H}_{2} \mathrm{O}\right] /[\mathrm{p}-$ $\left.\mathrm{H}_{2} \mathrm{O}\right]=3$. We find the $[\mathrm{HDO}] /\left[\mathrm{H}_{2} \mathrm{O}\right]$ ratio towards $\mathrm{SgrB} 2$, at 63 and $81 \mathrm{~km} \mathrm{~s}^{-1}$, to be $\sim 5 \times 10^{-4}$ and $\sim 10^{-3}$ respectively (see Table 4). These values are about 30 and 70 times higher than the measured deuterium abundance in the Local Interstellar Medium $\left(1.5 \times 10^{-5}\right.$, Linsky 1998). The enhancement of the deuterium fractionation observed in the $[\mathrm{HDO}] /\left[\mathrm{H}_{2} \mathrm{O}\right]$ ratio appears even more important if one considers that deuterium has been measured to be under-abundant in the Galactic Center region. Lubowich et al. (2000) have estimated the $[\mathrm{D}] /[\mathrm{H}]$ ratio towards SgrA to be around $1.7 \times 10^{-6}$. Similar values have been measured towards SgrB2 by Jacq et al. (1999) and by Polehampton et al. (2002). All estimates are affected by very large uncertainties, however an order-of-magnitude comparison with our measured $[\mathrm{HDO}] /\left[\mathrm{H}_{2} \mathrm{O}\right]$ ratio shows that it is actually a few $10^{2}$ times higher than the deuterium abundance in the region. This value, scaled to the lower $[\mathrm{D}] /[\mathrm{H}]$ ratio in the Galactic Center, is consistent with that predicted by the steady-state chemical models of Roberts \& Millar (2000) for gas temperatures ranging between $\sim 30$ and $100 \mathrm{~K}$ and densities between $\sim 10^{3}$ and $10^{8} \mathrm{~cm}^{-3}$, roughly the range of temperatures and densities expected in the warm envelope. However, the analysis presented in the next section shows that the HDO and $\mathrm{H}_{2} \mathrm{O}$ absorption are actually produced in different locations, hence the above mentioned value of the $[\mathrm{HDO}] /\left[\mathrm{H}_{2} \mathrm{O}\right]$ ratio has little relevance. We will address the issue again in Sect. 5 . 


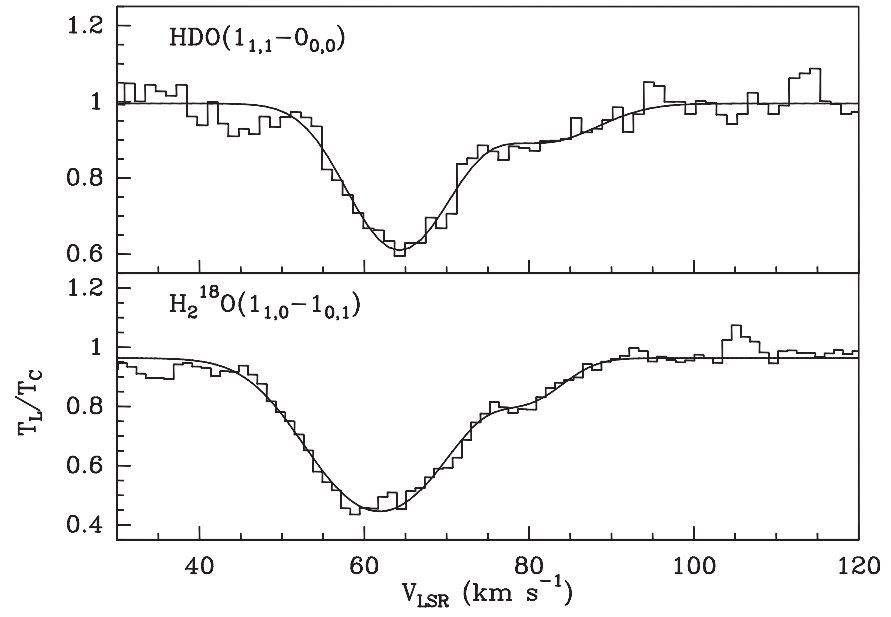

Fig. 2. The $894-\mathrm{GHz}$ HDO $\left(1_{1,1}-0_{0,0}\right)$ and the $548-\mathrm{GHz}$ o$\mathrm{H}_{2}^{18} \mathrm{O}\left(1_{1,0}-1_{0,1}\right)$ absorption features observed towards SgrB2. The spectrum in the upper panel is the sum of the scans relative to the HDO absorption towards SgrB2(M) and SgrB2(N) separately (see Fig. 1), observed at the CSO with a $10^{\prime \prime}$ beam. The lower panel shows the $\mathrm{o}-\mathrm{H}_{2}^{18} \mathrm{O}$ absorption towards SgrB2, observed by N00 with SWAS $\left(3.3 \times 4{ }^{\prime} .5\right.$ beam $)$. The $y$-scale gives the line-to-continuum $\left(T_{\mathrm{L}} / T_{\mathrm{C}}\right)$ ratio. The solid curves represent the Gaussian fits to the line opacity. The radial velocities, line opacities and widths, derived from the fit for both transitions, are listed in Table 3. The larger width of the SWAS $\mathrm{o}-\mathrm{H}_{2}^{18} \mathrm{O}$ line, with respect to the HDO feature resulting from the combination of the CSO scans, agrees with the extended water distribution observed by, e.g., Cernicharo et al. (1997) and Neufeld et al. (in prep.).

\section{Modeling the distribution of water}

As previously mentioned (Sect. 1), it is known from observations of, e.g., $\mathrm{H}_{2} \mathrm{CO}$ (cf. Whiteoak \& Gardner 1979; Martín-Pintado et al. 1990) that the two main cores in the SgrB2 complex - SgrB2(N) and SgrB2(M) - are embedded in a warm $\left(15 \mathrm{~K} \lesssim T_{\text {gas }} \lesssim 100 \mathrm{~K}\right)$, dense $\left(n\left(\mathrm{H}_{2}\right) \sim 10^{5} \mathrm{~cm}^{-3}\right)$ envelope of molecular gas. Also, a hot $\left(500 \mathrm{~K} \lesssim T_{\text {gas }} \lesssim 700 \mathrm{~K}\right)$ and diffuse $\left(10^{3} \mathrm{~cm}^{-3} \lesssim n\left(\mathrm{H}_{2}\right) \lesssim 10^{4} \mathrm{~cm}^{-3}\right)$ gas layer is located in the foreground, as indicated by the detection in absorption of high-energy ammonia transitions (Wilson et al. 1982; Hüttemeister et al. 1995; C02). Both gas components show characteristic radial velocities around 65 and $81 \mathrm{~km} \mathrm{~s}^{-1}$ (the latter being observable only towards the northern core), hence the hot foreground gas layer is thought to be physically connected to the cloud complex as well.

The interpretation of the results illustrated in Sect. 3.2 strongly depends on the assumptions about the location of the absorbing gas. Absorption due to molecules such as $\mathrm{H}_{2} \mathrm{O}$ and its isotopomers has generally been attributed to the warm envelope (Z95a; N00), under the assumption that only the groundstate level was populated. However, $\mathrm{C} 02$ have proposed that all the absorbing water might instead be located in the hot gas layer. In this case, according to $\mathrm{C} 02$, the estimated column density of $\mathrm{H}_{2} \mathrm{O}$ towards the main velocity components would be about one order of magnitude higher than that quoted, for example, by Z95a and by us in Sect. 3.2. Such a high value would support the C-shock model used by Flower and collaborators to explain the observed features of the hot layer, such as the high gas temperature, low gas density and limited spatial width (Flower et al. 1994; Flower et al. 1995).

From a chemical point of view, it is entirely possible that a large percentage of the observed water column density be located within the diffuse hot layer rather than in the warm envelope. In fact, the gas temperature in the hot layer is sufficient not only to induce evaporation of water ices from the surface of dust grains, but also to trigger the gas-phase production of $\mathrm{H}_{2} \mathrm{O}$ via the following chain of neutral-neutral reactions:

$\mathrm{O}+\mathrm{H}_{2} \rightarrow \mathrm{OH}+\mathrm{H}$

$\mathrm{OH}+\mathrm{H}_{2} \rightarrow \mathrm{H}_{2} \mathrm{O}+\mathrm{H}$,

which proceed very rapidly as $T \gtrsim 400 \mathrm{~K}$ (Elitzur \& de Jong 1978, also cf. Bergin et al. 1998). Therefore, we can expect the abundance of $\mathrm{H}_{2} \mathrm{O}$ to be enhanced in the hot layer with respect to the warm envelope, up to the point where all the oxygen not locked in $\mathrm{CO}$ is converted into $\mathrm{H}_{2} \mathrm{O}$. In fact, $\mathrm{HDO}$ can be formed in shocked gas as well, via the reactions (Bergin et al. 1999):

$\mathrm{O}+\mathrm{HD} \rightarrow \mathrm{OD}+\mathrm{H}$

$\mathrm{OD}+\mathrm{H}_{2} \rightarrow \mathrm{HDO}+\mathrm{H}$,

and

$\mathrm{OH}+\mathrm{HD} \rightarrow \mathrm{HDO}+\mathrm{H}$.

But, because of the lower HD abundance, the timescales are slower for these reactions than for the $\mathrm{H}_{2} \mathrm{O}$ production paths, such that all the available atomic oxygen will be locked in $\mathrm{H}_{2} \mathrm{O}$ prior to the formation of HDO. Thus, gas-phase chemistry in the hot layer yields $[\mathrm{HDO}] /\left[\mathrm{H}_{2} \mathrm{O}\right] \sim[\mathrm{D}] /[\mathrm{H}]$. We believe this picture cannot be significantly altered by grain chemistry, because $i$ ) the temperature of the dust in the hot layer is known to be much lower than that of the gas (cf. Wilson et al. 1982; Hüttemeister et al. 1993), too low to allow evaporation of ice mantles from the grain surface; $i$ ) sputtering of dust grains, on the other hand, cannot account for the release of intact molecules; and however, iii), the build-up of ice mantles is not efficient at low densities, hence the deposit of water ices on the grains surface is likely to be negligible in the first place $^{2}$.

To summarize, we believe that it is reasonable to expect the contribution of the hot layer to the observed HDO absorption to be negligible, whereas its contribution to the observed $\mathrm{H}_{2} \mathrm{O}$ absorption is likely to be significant. It is important to estimate the extent of such a contribution: a precise determination of the column density of water would set a tight constraint on the physical models that attempt to identify the heating mechanism responsible for the high temperatures in the hot layer. Unfortunately, since both gas components show roughly the same radial velocity, it is not possible to separate, by purely observational means, the ground-state water absorption produced in the warm envelope from that produced in the hot layer.

2 This is supported, for example, by observations of high abundances of gas-phase atomic oxygen in molecular clouds with density $\sim 10^{2}-10^{3} \mathrm{~cm}^{-3}$ (Lis et al. 2001), suggesting that much of the oxygen which is not locked in CO could well be present in the gas in its atomic form, rather than depleted on dust grains. 
Table 3. Results of the Gaussian fits to the optical depth of the HDO (Cols. 1-3) and o- $\mathrm{H}_{2}^{18} \mathrm{O}$ (Cols. 4-6, N00 data) absorption features observed towards SgrB2 and shown in Fig. 2. The listed parameters are, for each transition, respectively the radial velocity, the line opacity and the line width. The fits have been performed with the method ABSORPTION from the GILDAS CLASS package.

\begin{tabular}{|c|c|c|c|c|c|}
\hline \multicolumn{3}{|c|}{$\operatorname{HDO}\left(1_{1,1}-0_{0,0}\right)$} & \multicolumn{3}{|c|}{$\mathrm{o}-\mathrm{H}_{2}^{18} \mathrm{O}\left(1_{1,0}-1_{0,1}\right)$} \\
\hline $\begin{array}{c}v_{\mathrm{LSR}} \\
\left(\mathrm{km} \mathrm{s}^{-1}\right)\end{array}$ & $\tau$ & $\begin{array}{c}\Delta v \\
\left(\mathrm{~km} \mathrm{~s}^{-1}\right)\end{array}$ & $\begin{array}{c}v_{\mathrm{LSR}} \\
\left(\mathrm{km} \mathrm{s}^{-1}\right)\end{array}$ & $\tau$ & $\begin{array}{c}\Delta v \\
\left(\mathrm{~km} \mathrm{~s}^{-1}\right)\end{array}$ \\
\hline $64.3 \pm 0.3$ & $0.49 \pm 0.03$ & $13.3 \pm 0.7$ & $61.9 \pm 0.3$ & $0.77 \pm 0.02$ & $17.7 \pm 0.7$ \\
\hline $82.0 \pm 0.0$ & $0.11 \pm 0.02$ & $14.6 \pm 0.2$ & $80.3 \pm 0.9$ & $0.13 \pm 0.02$ & $9.6 \pm 2.7$ \\
\hline
\end{tabular}

Table 4. Total column densities of $\mathrm{HDO}$ and $\mathrm{o}-\mathrm{H}_{2}^{18} \mathrm{O}$ in the $3.3 \times 4.5$ SWAS beam, producing the absorption features shown in Fig. 2. Column 1: average radial velocity of the gas component (calculated from the values in Table 3, Cols. 1 and 4); Cols. 2 and 3: total column density of HDO and $\mathrm{o}-\mathrm{H}_{2}^{18} \mathrm{O}$ calculated using Eq. (1) and the fit parameters in Table 3. Since we assumed only the ground-state level the absorbing material to be populated, these are to be considered as lower limits. Column 4: $[\mathrm{HDO}] /\left[\mathrm{H}_{2} \mathrm{O}\right]$ ratio, calculated on the basis of the estimated column densities of $\mathrm{HDO}$ and $\mathrm{o}-\mathrm{H}_{2}^{18} \mathrm{O}$ assuming that $\left[{ }^{16} \mathrm{O}\right] /\left[{ }^{18} \mathrm{O}\right]=261 \pm 20\left(\right.$ Whiteoak \& Gardner 1981) and that $\left[\mathrm{o}-\mathrm{H}_{2} \mathrm{O}\right] /\left[\mathrm{p}-\mathrm{H}_{2} \mathrm{O}\right]=3$.

\begin{tabular}{ccccc}
\hline \hline $\begin{array}{c}v_{\text {LSR }} \\
\left(\mathrm{km} \mathrm{s}^{-1}\right)\end{array}$ & $\begin{array}{c}N(\mathrm{HDO}) \\
\left(10^{13} \mathrm{~cm}^{-2}\right)\end{array}$ & $\begin{array}{c}N\left(\mathrm{o}-\mathrm{H}_{2}^{18} \mathrm{O}\right) \\
\left(10^{13} \mathrm{~cm}^{-2}\right)\end{array}$ & $\begin{array}{c}N\left(\mathrm{H}_{2} \mathrm{O}\right) \\
\left(10^{16} \mathrm{~cm}^{-2}\right)\end{array}$ & $\begin{array}{c}{[\mathrm{HDO}] /\left[\mathrm{H}_{2} \mathrm{O}\right]} \\
\left(10^{-3}\right)\end{array}$ \\
\hline$\sim 63$ & $1.67 \pm 0.13$ & $6.33 \pm 0.30$ & $2.20 \pm 0.20$ & $0.8 \pm 0.1$ \\
$\sim 81$ & $0.40 \pm 0.07$ & $0.60 \pm 0.19$ & $0.21 \pm 0.07$ & $1.9 \pm 0.7$ \\
\hline
\end{tabular}

However, a number of $\mathrm{HDO}$ and $\mathrm{H}_{2}^{18} \mathrm{O}$ transitions have been observed towards the SgrB2 cores with a variety of instruments (see Table 5 and Fig. 3), and detailed modeling can be performed to disentangle the contribution to the observed features from the different cloud components.

The available dataset, enriched by our detection of the ground-state HDO transition at $894 \mathrm{GHz}$, provides sufficient observational constraints to model, in a self-consistent manner, the $\mathrm{HDO}$ and $\mathrm{H}_{2} \mathrm{O}$ abundance in all three components of the SgrB2 cloud. In detail:

a) the observed emission lines of $\mathrm{p}-\mathrm{H}_{2}^{18} \mathrm{O}$ at $203 \mathrm{GHz}$ (SgrB2(N), Gensheimer et al. 1996), and of HDO at 143, 226, $241 \mathrm{GHz}$ (SgrB2(M) and N, Jacq et al. 1990) constrain the HDO and water abundance and the $\mathrm{H}_{2}$ density in the hot-core-type components;

b) being virtually absent in the hot layer, HDO can be used to calibrate the water content of the warm envelope. In particular, the 894-GHz HDO absorption feature (this work) observed towards both $\operatorname{SgrB} 2(\mathrm{M})$ and $\mathrm{N}$, together with the $[\mathrm{HDO}] /\left[\mathrm{H}_{2} \mathrm{O}\right]$ ratio estimated at point $a$ ), help us set the $\mathrm{HDO}$ and $\mathrm{H}_{2} \mathrm{O}$ abundance in this region;

c) finally, having estimated the column density of water in the warm envelope, it is possible to assess whether or not the quantity of non-deuterated water in the warm envelope can be responsible for the $\mathrm{H}_{2}^{18} \mathrm{O}$ absorption observed at $548 \mathrm{GHz}$ (N00, Z95a). The $180-\mu \mathrm{m} \mathrm{o}-\mathrm{H}_{2}^{18} \mathrm{O}$ line observed by Cernicharo et al. (1997) towards the SgrB2 complex is blended with the $\mathrm{H}_{3} \mathrm{O}^{+}\left(1_{1}^{-}-1_{1}^{+}\right)$line (Goicoechea \& Cernicharo 2001), and cannot therefore be used as a reference for our purposes.

\subsection{The model}

We use the static radiative transfer code described by Zmuidzinas et al. (1995b) (hereafter Z95b) to reproduce the intensities observed for the features listed in Table 5. A few changes have been made with respect to the original version, but the bulk of the model is the same and can be summarized as follows:

- A background, internally heated continuum source is embedded in a spherical molecular envelope of radius $0.05 \mathrm{pc} \leq r \leq 22.5 \mathrm{pc}$ (corresponding to the warm envelope as defined at the beginning of Sect. 4).

- The large-scale structure of the cloud is well constrained by the observations of $\mathrm{C}^{18} \mathrm{O}$ carried out by Lis \& Goldsmith (1989, hereafter LG89). The density profile adopted in the model, as well as the radial variation of the dust temperature, correspond to their Model C. The temperature of the gas is coupled to that of the dust, $T_{\text {dust }}=T_{\text {gas }}=T$.

- The abundance of the modeled species, assumed to be constant throughout the envelope, is a free parameter, as well as the width (in $\mathrm{km} \mathrm{s}^{-1}$ ) of the modeled feature.

- The code allows radiative transfer calculations for one Gaussian velocity component only. In what follows, we will only try to reproduce the $65-\mathrm{km} \mathrm{s}^{-1}$ component of the molecular gas.

This geometrically straightforward model has proved successful in reproducing the dust continuum emission observed towards the SgrB2 cloud complex at $\mathrm{mm}$ and submm wavelengths, as well as absorption features such as the $\mathrm{HCl}(1-0)$ and $\mathrm{H}_{2}^{18} \mathrm{O}\left(1_{1,0}-1_{0,1}\right)$ lines observed in the submm band with the Kuiper Airborne Observatory (KAO, see Z95a and Z95b). Nevertheless, it is not capable, as it is, to reproduce those HDO and $\mathrm{H}_{2}^{18} \mathrm{O}$ features that have been observed in emission, and that are thought to be produced in the hot-core-type components of SgrB2(M) and SgrB2(N) (cf. Jacq et al. 1990; Gensheimer et al. 1996). In fact, a molecular hot-core component, intended as a compact, dense, warm molecular region characterized by fairly flat radial profiles of density and temperature, is missing in the model, since the region of radius $r<0.05 \mathrm{pc}$ is devoid of molecular gas. A radius of 
Table 5. Data set used to model the HDO and $\mathrm{H}_{2} \mathrm{O}$ distribution in the SgrB2 cloud complex. References are: Jacq et al. (1990) (J90), Neufeld et al. (2000) (N00), Gensheimer et al. (1996) (G96). The set of HDO transitions is displayed in Fig. 3.

\begin{tabular}{lrcccl}
\hline \hline Transition & $\begin{array}{r}E_{1} / k \\
(\mathrm{~K})\end{array}$ & $\begin{array}{c}\text { Frequency } \\
(\mathrm{GHz})\end{array}$ & HPBW & $\begin{array}{c}\text { Observed } \\
\text { towards }\end{array}$ & Ref. \\
\hline $\mathrm{HDO}\left(1_{1,1}-0_{0,0}\right)^{a}$ & 0.0 & 893.6 & $11^{\prime \prime}$ & $\mathrm{M}, \mathrm{N}$ & this work (CSO) \\
$\mathrm{HDO}\left(2_{1,1}-2_{1,2}\right)^{b}$ & 83.6 & 241.6 & $10^{\prime \prime}$ & $\mathrm{N}$ & J90 (IRAM 30 m) \\
$\mathrm{HDO}\left(3_{1,2}-2_{2,1}\right)^{b}$ & 156.7 & 225.9 & $11^{\prime \prime}$ & $\mathrm{M}, \mathrm{N}$ & J90 (IRAM 30 m) \\
$\mathrm{HDO}\left(4_{2,2}-4_{2,3}\right)^{b}$ & 312.3 & 143.7 & $17^{\prime \prime}$ & $\mathrm{N}$ & J90 (IRAM 30 m) \\
$\mathrm{o}-\mathrm{H}_{2}^{18} \mathrm{O}\left(1_{1,0}-1_{0,1}\right)^{a}$ & 34.2 & 547.7 & $3 \prime 9$ & $\mathrm{M}+\mathrm{N}$ & N00 (SWAS) \\
$\mathrm{p}-\mathrm{H}_{2}^{18} \mathrm{O}\left(3_{1,3}-2_{2,0}\right)^{b}$ & 193.9 & 203.4 & $12^{\prime \prime}$ & $\mathrm{N}$ & G96 (IRAM 30 m) \\
\hline
\end{tabular}

\footnotetext{
${ }^{a}$ Absorption.

${ }^{b}$ Emission.
}

$\sim 0.05 \mathrm{pc}$ has indeed been attributed to the hot cores embedded in SgrB2(M) and N, based on the high-spatial-resolution interferometric maps of the continuum emission towards the complex (cf. Lis et al. 1993; hereafter L93). Therefore, we have modified the model by introducing a region of radius $r<0.05 \mathrm{pc}$, in which the $\mathrm{H}_{2}$ density and the gas and dust temperatures are constant and equal the values of density and temperature at $r=0.05 \mathrm{pc}$, based on the radial profiles of LG89, Model C. This yields $n\left(\mathrm{H}_{2}\right) \sim 3.4 \times 10^{7} \mathrm{~cm}^{-3}$ and $T_{\text {dust }}=T_{\text {gas }} \sim 200 \mathrm{~K}$ in the hot-core component. Both values are in good agreement with the estimates based on the continuum emission $\left(n\left(\mathrm{H}_{2}\right) \sim 3 \times 10^{7} \mathrm{~cm}^{-3}\right.$ in the hot core of SgrB2(M), and $n\left(\mathrm{H}_{2}\right) \sim 2 \times 10^{7} \mathrm{~cm}^{-3}$ in SgrB2(N), L93) and on the intensities of metastable and non-metastable ammonia lines ( $T_{\text {kin }} \sim T_{\text {rot }}=202 \pm 15 \mathrm{~K}$ in SgrB2(M), Vogel et al. 1987). Note that the introduction of the tiny hot-core component does not produce significant variations in the predicted continuum emission.

We also consider the effect of temperature on the gas-phase abundance of the modeled species. A vast $(\sim 1.7 \mathrm{pc} \leq r \leq$ $22.5 \mathrm{pc}$ ) portion of the warm envelope shows temperatures lower than $100 \mathrm{~K}$, which favour the freeze-out of gas-phase water onto dust grains (cf. Williams 1993 and references therein). The abundance of water (both deuterated and non-deuterated) in this region will naturally be lower than it would be at temperatures higher than $100 \mathrm{~K}$, and particularly in the vicinity of the hot cores, where the higher $T$ promotes the evaporation of water ices from the grains surface. The second major modification to the $\mathrm{Z95b}$ model involves therefore a differentiation between a warmer $\left(T>100 \mathrm{~K}\right.$, hereafter Phase $\left.\mathrm{I}^{3}\right)$ and a colder $(T<100 \mathrm{~K}$, hereafter Phase II) region, identified by different gas-phase abundances. This is of course an oversimplification, however such an approximation is sufficient to reproduce the data correctly, within the errors, as will be illustrated in Sect. 4.2. As already discussed by Neufeld et al. (1997), who introduced a similar differentiation in order to reproduce the $\mathrm{H}_{2} \mathrm{O}\left(4_{3,2}-4_{2,3}\right)$ emission line observed with ISO at $122 \mu \mathrm{m}$, this freeze-out assumption is required by the observations: a reduction of the $\mathrm{HDO}$ and $\mathrm{H}_{2} \mathrm{O}$ abundance in the outer regions of the envelope is necessary to model the $\mathrm{HDO}$ and $\mathrm{H}_{2}^{18} \mathrm{O}$

\footnotetext{
${ }^{3}$ Note that Phase I includes, but is not limited to, the hot-core component of the cloud (see sketch in Fig. 4).
}

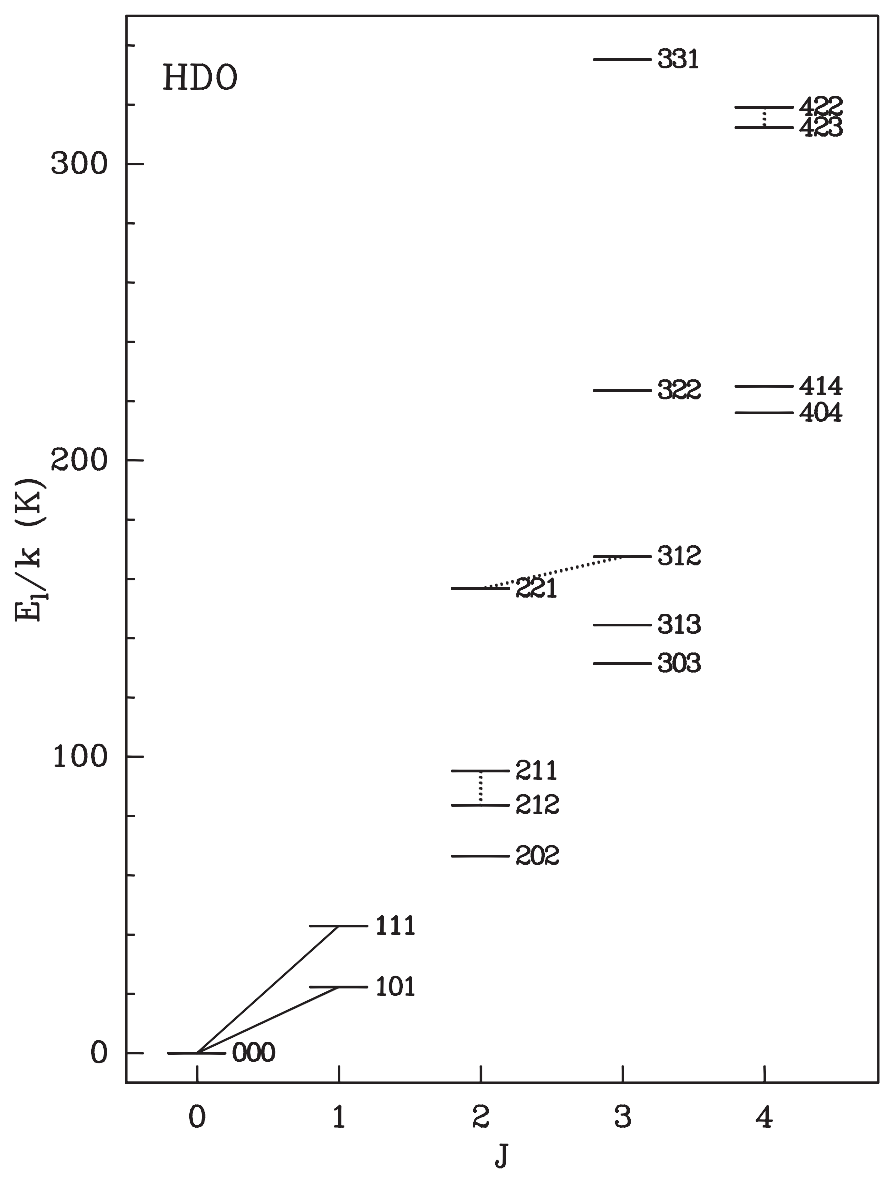

Fig. 3. Rotational levels of deuterated water up to $\sim 400 \mathrm{~K}$. The transitions included in our data set are indicated (solid lines: this work; dotted lines: Jacq et al. 1990; see Table 5).

emission radiated from the hot-core gas and, at the same time, the HDO absorption thought to be produced in the warm envelope. In fact, a decrease in the water abundance towards the outer regions several molecular cloud cores has been observed by Snell et al. (2000b). The sketch in Fig. 4 shows the relative sizes of hot core and warm envelope, as well as of the Phase I and Phase II regions.

Finally, as indicated in Table 5, all the features in our sample but the two $\mathrm{o}-\mathrm{H}_{2}^{18} \mathrm{O}$ lines have been observed with spatial resolutions ranging between $10^{\prime \prime}$ and $17^{\prime \prime}$. Since the projected 
distance between the northern and the middle core is of $\sim 47^{\prime \prime}$ ( $\sim 2 \mathrm{pc}$ at a distance of $8.5 \mathrm{kpc}$ ), it is more appropriate to model the two cores separately. In fact, $\mathrm{M}$ and $\mathrm{N}$ show different chemical compositions, with the northern core displaying higher abundances of complex molecules (cf. Snyder et al. 1994; Miao \& Snyder 1997 and references therein; Nummelin et al. 2000). The middle core, on the other hand, presents a higher mass and luminosity, as inferred from the analysis of the dust emission at several wavelengths (cf. Goldsmith et al. 1992; L93; Gordon et al. 1993; Vogel et al. 1987; Dowell et al. 1999). Such dissimilarities seem to reflect the youth of $\mathrm{N}$ relative to $\mathrm{M}$. However, for our purposes we will neglect the chemical differences between the two cores, since the absence of molecular species in the middle core mostly concerns large complex molecules (see references above). Moreover, our simple model shows that the different continuum temperatures observed towards the two cores can be reproduced fairly well by simply assuming different dust properties for the two cores. L93 suggested, for the grain emissivity law in the northern core, a shallower slope than that of the middle core, a difference that may be explained by different grain shapes in the two cores.

We proceed by running our radiative transfer model separately for the two sources. The results will be appropriately combined, when necessary, to be compared to the data (see Sect. 4.2). We assume identical physical parameters (density and temperature profiles, size of the hot core and of the warm envelope, $\mathrm{H}_{2} \mathrm{O}$ and $\mathrm{HDO}$ abundance) for both $\mathrm{M}$ and $\mathrm{N}$. The only difference between the two cores lies in the grain emissivity law, that has a slope of 1.1 in the northern core, and of 1.4 in the middle one (L93). This condition holds only in the inner $\sim 1 \mathrm{pc}$ of the cloud, which roughly correspond to half the projected distance between the two cores. In fact, we must take into account the fact that the warm envelopes in which the cores are embedded will, at some point, merge to form one single "shared" envelope. Hence, for radii larger than half the distance between the cores, we assume the dust properties to be the same, with a grain emissivity slope of 1.5 as derived from submm flux densities in a 60" beam (Goldsmith et al. 1990).

\section{2. $\mathrm{HDO}$ and $\mathrm{H}_{2} \mathrm{O}$ in the hot cores and in the warm envelope}

We aim at reproducing the 143-, 226-, and 241-GHz HDO emission features, observed by Jacq et al. (1990, also see Table 5), in order to model the gas-phase abundance of this species in the hot-core components of SgrB2(M) and N. At the same time, we rely on the $894-\mathrm{GHz}$ ground-state absorption feature (Fig. 1) to constrain the HDO abundance in the warm envelope. The closest match between model and data is obtained when the abundance of HDO, relative to $\mathrm{H}_{2}$, is set to $1.5 \times 10^{-9}$ in Phase I, and $3.5 \times 10^{-11}$ in Phase II of the molecular cloud, i.e., a depletion of a factor of 40 is observed in Phase II. We note that this model predicts the 465-GHz line to be in weak emission, in agreement with the observations (cf. Sect. 2). The total column density of HDO in a $10^{\prime \prime}$ beam, $N(\mathrm{HDO})$, is as high as $2 \times 10^{16} \mathrm{~cm}^{-2}$, almost three orders of magnitude higher than estimated from the $894-\mathrm{GHz}$ absorption line (Sect. 3.1) under the assumption that all the absorbing HDO is in the ground state. This inconsistency is explained by the radial distribution of the fractional populations of the first three levels of $\operatorname{HDO}\left(0_{0,0}, 1_{0,1}\right.$ and $1_{1,1}$ in order of increasing energy, Fig. 5): the fractional population of the ground level exceeds $90 \%$ for $r \gtrsim 3$ pc only, hence our calculations in Sect. 3.1 severely underestimate the total HDO column density in the innermost portion of the cloud, which shows the highest gas density and water abundance and thus contributes the largest percentage ( $\geq 99 \%)$ of the total HDO column density. Incidentally, note that our value of $N(\mathrm{HDO})$ is only about one order of magnitude higher than that estimated by Jacq et al. (1990) towards SgrB2(N) through the analysis of the hot-core transitions, that instead trace the densest gas in the complex.

The abundance of non-deuterated water in the hot core can be modeled on the basis of the $203-\mathrm{GHz}$ p- $\mathrm{H}_{2}^{18} \mathrm{O}$ transition observed, in emission, towards SgrB2(N) (Gensheimer et al. 1996). We are able to reproduce the measured intensity of this line if we assume the abundance of $\mathrm{p}-\mathrm{H}_{2}^{18} \mathrm{O}$ to be $2.2 \times 10^{-9}$ in Phase I. Note that this feature shows, towards SgrB2(N), severe blending with a wide $\mathrm{SO}_{2}$ line, therefore the intensity indicated by Gensheimer et al. should be taken cum grano salis. However, a variation of the measured intensity of the $203-\mathrm{GHz}$ line up to $50 \%$ will not introduce significant changes in our fit results.

Assuming an ortho/para ratio of 3 , and a $\left[{ }^{16} \mathrm{O}\right] /\left[{ }^{18} \mathrm{O}\right]$ ratio of $261 \pm 20$ (Whiteoak \& Gardner 1981), we estimate the $\mathrm{H}_{2} \mathrm{O}$ abundance to be $\sim 2.3 \times 10^{-6}$ in this region. This result yields a $[\mathrm{HDO}] /\left[\mathrm{H}_{2} \mathrm{O}\right]$ ratio of $6.5 \times 10^{-4}$, which is in order-of-magnitude agreement with the estimate of $1.8 \times 10^{-4}$ by Gensheimer et al. (1996). The discrepancy between the $[\mathrm{HDO}] /\left[\mathrm{H}_{2} \mathrm{O}\right]$ ratio calculated by us, and the $[\mathrm{D}] /[\mathrm{H}]$ ratio measured towards the Galactic Center (cf. Lubowich et al. 2000), will be discussed in Sect. 5 .

Knowing the $[\mathrm{HDO}] /\left[\mathrm{H}_{2} \mathrm{O}\right]$ ratio, the $\mathrm{o}-\mathrm{H}_{2}^{18} \mathrm{O}$ abundance in Phase II can be determined ${ }^{4},\left[0-\mathrm{H}_{2}^{18} \mathrm{O}\right] \sim 2.1 \times 10^{-10}$. The resulting total peak column density of $\mathrm{H}_{2} \mathrm{O}$ (hot core + warm envelope) is $\sim 3.6 \times 10^{19} \mathrm{~cm}^{-3}$. The $548-\mathrm{GHz}$ o- $\mathrm{H}_{2}^{18} \mathrm{O}$ absorption expected to be produced in the warm envelope is then predicted, for each core, with our radiative transfer code. The two spectra are opportunely combined to reproduce the attenuation of the SWAS beam, and finally compared to the N00 data.

A quantitative comparison between the measured intensities of the whole set of HDO and $\mathrm{H}_{2}^{18} \mathrm{O}$ lines and the model results can be found in Table 6. Most of the spectral line data

\footnotetext{
4 We can reasonably assume that the gas-phase water abundance at this stage be determined mostly by evaporation from dust grains or freeze-out onto dust grains, and that these processes affect both $\mathrm{H}_{2} \mathrm{O}$ and HDO equally. In other words, we assume the $[\mathrm{HDO}] /\left[\mathrm{H}_{2} \mathrm{O}\right]$ ratio to be constant throughout Phases I and II. However, even considering that fractionation is likely to take place in the cold portions of the gas, the $[\mathrm{HDO}] /\left[\mathrm{H}_{2} \mathrm{O}\right]$ ratio in the Galactic Center is expected to assume a maximum value of $\sim 10^{-3}$ (E. Bergin, priv. comm.; also cf. Roberts \& Millar 2000), not very far from our estimate and therefore not affecting our conclusions. To make our point even stronger, fractionation will be most active in the coldest, outermost regions of the envelope, which contribute less than $1 \%$ to the total gas column density.
} 


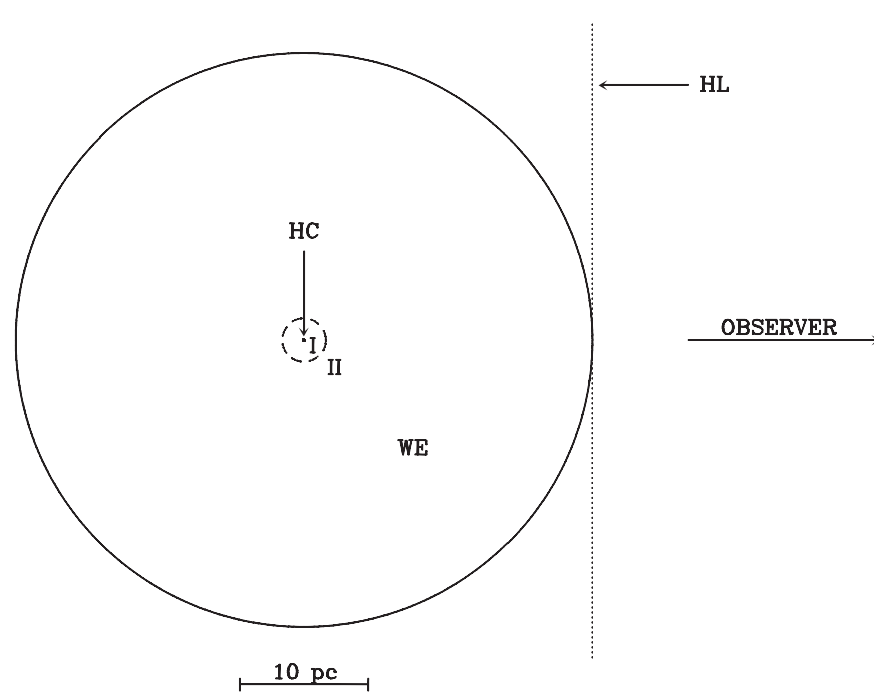

Fig. 4. Sketch illustrating the relative sizes of the SgrB2 cloud components. For practical reasons, only one of the two cores is represented here. The hot core (labelled HC in the figure) has a radius of $0.05 \mathrm{pc}$, and is tiny compared to the size of the warm envelope (WE, $r=22.5 \mathrm{pc}$ ). The dashed circle identifies the transition radius $(r=1.7 \mathrm{pc})$ between Phase I $(T>100 \mathrm{~K})$ and Phase II $(T<100 \mathrm{~K})$. Modeling of the spherically symmetric regions is illustrated in detail in Sect. 4.1. The hot layer (HL) is shown as a thin ( $0.02 \mathrm{pc}$ wide) sheet of gas lying right outside the warm envelope (see Sect. 4.3).

are reproduced within errors of $\sim 30 \%$, with three exceptions (indicated with a star in Table 6):

1) The 226-GHz HDO emission line observed towards $\mathrm{SgrB} 2(\mathrm{M})$ is a factor of 2 weaker, and

2) the $143-\mathrm{GHz}$ HDO line in $\mathrm{SgrB} 2(\mathrm{~N})$ is 4 times stronger than predicted by the model. Such discrepancies are likely to be due to the intrinsic chemical and physical differences between the molecular gas components of the two hot cores (cf. Miao \& Snyder 1997 and references therein), which have not been taken into account in our model.

3) Most interestingly, the predicted ground-state $\mathrm{o}-\mathrm{H}_{2}^{18} \mathrm{O}$ absorption at $548 \mathrm{GHz}$ is much shallower than observed (Fig. 2; N00): the abundance of non-deuterated water in the warm envelope is not sufficient ${ }^{5}$ to produce the absorption feature observed at $548 \mathrm{GHz}$.

\subsection{A "hot" issue: $\mathrm{H}_{2} \mathrm{O}$ in the hot layer}

The result that the observed $\mathrm{H}_{2}^{18} \mathrm{O}$ absorption cannot, according to our model, be produced in the warm envelope alone, is a very important one: first of all, our predictions confirm, at least from a qualitative point of view, the hypothesis of $\mathrm{C} 02$ that the most important contribution to the observed $\mathrm{H}_{2}^{18} \mathrm{O}$ absorption comes from the foreground hot gas layer. Secondly, having predicted the contribution of the warm envelope to the $\mathrm{H}_{2}^{18} \mathrm{O}$ absorption, it is possible to estimate the spatial width and

${ }^{5}$ In fact, the column density of $\mathrm{o}-\mathrm{H}_{2}^{18} \mathrm{O}$ is of the same order of magnitude as that of HDO, but the Einstein B coefficient of the o$\mathrm{H}_{2}^{18} \mathrm{O}\left(1_{1,0}-1_{0,1}\right)$ transition is about a factor of 2 smaller than that of the $\operatorname{HDO}\left(1_{1,1}-0_{0,0}\right)$ transition, resulting in a shallower absorption line.

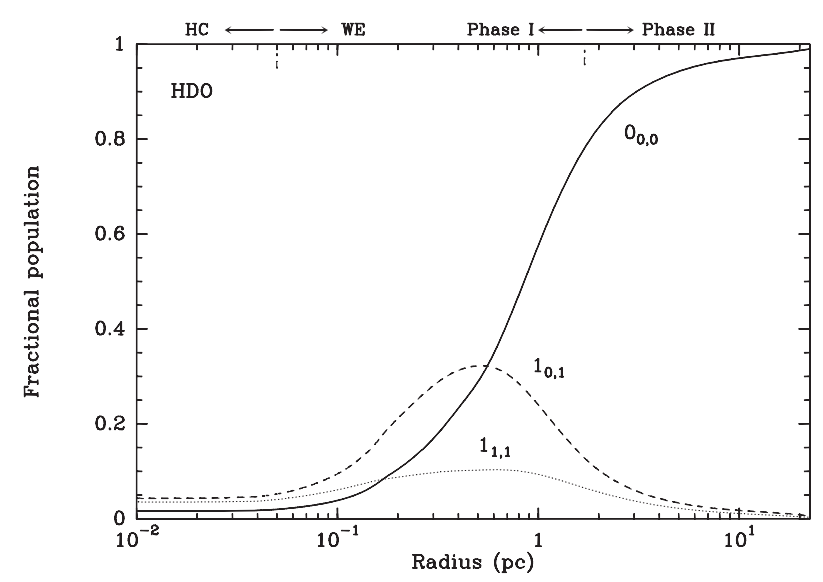

Fig. 5. Fractional populations of the three lowest-energy levels of the $\mathrm{HDO}$ as a function of the radius of the cloud.

Table 6. Comparison between the measured intensities and opacities of the $\mathrm{HDO}$ and $\mathrm{H}_{2}^{18} \mathrm{O}$ lines in our sample (Table 5), and the predictions from the modified Z95b model (Sect. 4). At this stage, only the hot cores and the warm envelope of SgrB2 are included in the model. Note that the predicted optical depth of the $548-\mathrm{GHz} \mathrm{o}-\mathrm{H}_{2}^{18} \mathrm{O}$ line is a factor of 8 smaller than observed (see Sects. 4.2 and 4.3).

\begin{tabular}{cccl}
\hline \hline $\begin{array}{c}\text { Line } \\
\text { (emission) }\end{array}$ & Source & $\begin{array}{c}\text { Observed } T_{\mathrm{mb}} \\
(\mathrm{K})\end{array}$ & $\begin{array}{l}\text { Model } T_{\mathrm{mb}} \\
(\mathrm{K})\end{array}$ \\
\hline $\mathrm{HDO} 241$ & $\mathrm{M}$ & - & 0.9 \\
& $\mathrm{~N}$ & 1.2 & 0.9 \\
$\mathrm{HDO} 226$ & $\mathrm{M}$ & 0.4 & $0.8^{\star}$ \\
& $\mathrm{N}$ & 1.3 & 0.8 \\
$\mathrm{HDO} 143$ & $\mathrm{M}$ & - & 0.1 \\
& $\mathrm{~N}$ & 0.4 & $0.1^{\star}$ \\
$\mathrm{p}-\mathrm{H}_{2}^{18} \mathrm{O} 203$ & $\mathrm{~N}$ & 1.5 & 1.5 \\
\hline (absorption) & & Observed $\tau$ & Model $\tau$ \\
$\mathrm{HDO} 894$ & $\mathrm{M}$ & 0.4 & 0.5 \\
& $\mathrm{~N}$ & 0.8 & 0.6 \\
$\mathrm{o}-\mathrm{H}_{2}^{18} \mathrm{O} 548$ & $\mathrm{M}+\mathrm{N}$ & 0.8 & $0.1^{\star}$ \\
\hline
\end{tabular}

^ Deviates by more than $50 \%$ from observed value.

$\mathrm{H}_{2} \mathrm{O}$ abundance necessary for the hot layer to produce the observed feature, given the observational constraints mentioned in Sect. 4. In what follows, we will assume the hot layer to be located right outside of the warm envelope (see Fig. 4), although its exact location does not matter for our purposes.

Because there is no reason to believe it has spherical symmetry, the hot layer cannot be modeled directly with our radiative transfer code. However, we can use the radiative transport equation to calculate the total intensity emerging from the hot layer, $I_{\mathrm{TOT}}$, given a background emission $I_{\mathrm{HC}+\mathrm{WE}}$ as calculated by our model for the complex made up of hot core and warm envelope (results listed in Table 6):

$I_{\mathrm{TOT}}=I_{\mathrm{HC}+\mathrm{WE}} \cdot \mathrm{e}^{-\tau_{\mathrm{HL}}}+I_{\mathrm{HL}} \cdot\left(1-\mathrm{e}^{-\tau_{\mathrm{HL}}}\right)$,

where $\tau_{\mathrm{HL}}$ is the optical depth of the hot layer, and $I_{\mathrm{HL}} \cdot(1-$ $\left.\mathrm{e}^{-\tau_{\mathrm{HL}}}\right)$ is the hot layer emission corrected for self-absorption. We assume a water abundance, relative to $\mathrm{H}_{2}$, of $8 \times 10^{-4}$, based on the abundance of atomic oxygen not locked in $\mathrm{CO}$ (cf. Meyer et al. 1998 for a determination of the interstellar O 
abundance; Shaver et al. 1983 and Rolleston et al. 2000 for the parametrization of the abundance gradient of carbon and oxygen in the Galaxy). The spatial width of the hot layer, $\Delta w_{\mathrm{HL}}$, is treated as a free parameter, with $0.01 \mathrm{pc} \leq \Delta w_{\mathrm{HL}} \leq 3 \mathrm{pc}$, and we assume the gas sheet to be extended perpendicularly to the line of sight (see Fig. 4).

Both dust and gas contribute to $\tau_{\mathrm{HL}}$ and $I_{\mathrm{HL}}$. However, it is reasonable to assume the contribution of such a thin, diffuse layer of dust to be negligible with respect to the background envelope, and only the emission and opacity of the molecular gas will be taken into account. The fractional populations of levels $1_{1,0}$ and $1_{0,1}$ of $\mathrm{o}-\mathrm{H}_{2}^{18} \mathrm{O}$ have been calculated, with our radiative transfer code, for a thin sheet of hot gas of density $n\left(\mathrm{H}_{2}\right)_{\mathrm{HL}}=10^{3} \mathrm{~cm}^{-3}$ and temperature $T_{\text {gas }}=500 \mathrm{~K}$. Both values match the lower limits derived by $\mathrm{C} 02$ through the modeling of the ammonia absorption features observed with ISO at infrared wavelengths. A strict upper limit on the $\mathrm{H}_{2}$ density, $n\left(\mathrm{H}_{2}\right)_{\mathrm{HL}} \lesssim 5 \times 10^{3} \mathrm{~cm}^{-3}$, has been determined by Hüttemeister et al. (1995) through cm-wavelength observations of $\mathrm{NH}_{3}, \mathrm{SiO}$ and $\mathrm{HC}_{3} \mathrm{~N}$. These values are further supported by measurements of the intensity of the 691-GHz $\mathrm{CO}(6-5)$ line, performed with the CSO by P. Schilke \& D.C. Lis (unpublished data). Also, note that the ratio of the fractional populations of the o$\mathrm{H}_{2}^{18} \mathrm{O}$ levels is insensitive to temperature changes in the 500 $700 \mathrm{~K}$ window indicated by $\mathrm{C} 02$.

The best fit of the $\mathrm{o}-\mathrm{H}_{2}^{18} \mathrm{O}$ column density is determined based on the distribution of the $\chi^{2}$ values given by:

$\chi^{2}=\frac{\left(\tau_{\mathrm{obs}}-\tau_{\mathrm{mod}}\right)^{2}}{\sigma\left(\tau_{\mathrm{obs}}\right)^{2}}$,

where $\tau_{\text {obs }}$ and $\tau_{\text {mod }}$ are, respectively, the opacity of the observed 548- $\mathrm{GHz} \mathrm{H}_{2}^{18} \mathrm{O}$ absorption, and the predicted opacity of the feature for each pair of $\left[\mathrm{H}_{2}^{18} \mathrm{O}\right]_{\mathrm{HL}}$ and $\Delta w_{\mathrm{HL}}$ values. $\sigma\left(\tau_{\mathrm{obs}}\right)$ is the error on the observed line opacity, calculated by adopting a $20 \%$ error on the continuum level measured by SWAS at $548 \mathrm{GHz}$ (N00). The minimum $\chi^{2}$ values yield a o- $\mathrm{H}_{2}^{18} \mathrm{O}$ peak column density of $\sim 10^{14} \mathrm{~cm}^{-2}$, hence a $\mathrm{H}_{2} \mathrm{O}$ column density of $\sim 3.5 \times 10^{16} \mathrm{~cm}^{-2}$, corresponding to $\Delta w_{\mathrm{HL}} \sim 0.02 \mathrm{pc}$. We consider the value of $\Delta w_{\mathrm{HL}}$ to be a lower limit, but expect it to be correct within a factor of a few $(\sim 2-3)$. If we assume a $[\mathrm{HDO}] /\left[\mathrm{H}_{2} \mathrm{O}\right]$ ratio equal to the $[\mathrm{D}] /[\mathrm{H}]$ ratio measured towards the Galactic Center $\left(1.7 \times 10^{-6}\right.$, Lubowich et al. 2000) as expected for gas at this temperature, then the HDO peak column density in the hot layer, $N(\mathrm{HDO})_{\mathrm{HL}} \sim 5.2 \times 10^{10} \mathrm{~cm}^{-2}$, will contribute with $0.3 \%$ only to the absorption produced by the HDO component located in the warm envelope, in agreement with our hypothesis (Sect. 4).

An overview on the radial density and temperature profiles adopted to model the three regions of the cloud complex is given in Table 7. Each profile, $P(r)$, is described by the equation

$P(r)=a_{0}+a_{1} \cdot\left(\frac{r}{r_{0}}\right)^{\alpha}$.

Also, Table 8 summarizes the peak column densities of $\mathrm{H}_{2}$, $\mathrm{HDO}$ and $\mathrm{H}_{2} \mathrm{O}$ resulting from our model. The $\mathrm{H}_{2} \mathrm{O}$ abundance and the $[\mathrm{HDO}] /\left[\mathrm{H}_{2} \mathrm{O}\right]$ ratio are also stated.

\section{Discussion}

The unambiguous identification of the hot layer as the gas component responsible for the $65-\mathrm{km} \mathrm{s}^{-1}$ water absorption towards SgrB2 is definitely the main result of this work. This confirms the general picture proposed by $\mathrm{C} 02$. However, our model provides, for the hot layer, a value of the water column density, $N\left(\mathrm{H}_{2} \mathrm{O}\right)_{\mathrm{HL}}$, which is a factor of $\sim 10$ lower than that inferred by $\mathrm{C} 02$, due to the fact that we assumed a lower $\mathrm{H}_{2}$ density. In fact, previous estimates of the column density of the absorbing water (cf. Z95a, N00, and Sect. 3.2 of this paper) are in very good agreement with our result of $N\left(\mathrm{H}_{2} \mathrm{O}\right)_{\mathrm{HL}} \sim 3.5 \times 10^{16} \mathrm{~cm}^{-2}$ : the assumption employed in these estimates that only the ground state of $\mathrm{H}_{2} \mathrm{O}$ be populated is correct for the hot layer, although it does not apply to the warm envelope. However, the column density of water actually responsible for the observed absorption is negligible, with respect to the total column density estimated over the whole envelope (see Table 8). It is important to keep in mind that column densities calculations from groundstate absorption features, if based on the "classical" assumptions mentioned in Sect. 3.1, can be highly misleading in regions as complex as SgrB2, and are likely to yield underestimates of several orders of magnitude (a factor of $\sim 1000$ in our case).

As previously mentioned, several heating mechanism have been proposed to explain the existence of such a hot, relatively thin sheet of molecular gas. The origin of the whole cloud complex has been proposed to be linked to large-scale cloud-cloud collisions (Hasegawa et al. 1994), so shock-induced heating is definitely possible, although it remains to be explained why the velocity of the hot layer is identical to that of the warm envelope. Martín-Pintado et al. (2000) argue rather in favour of an X-ray-driven chemistry, based on the observed spatial correlation of the $\mathrm{Fe} 6.4-\mathrm{keV}$ emission line with the $\mathrm{SiO}(1-$ 0 ) emission at $43.4 \mathrm{GHz}$. In a recent paper by Goicoechea \& Cernicharo (2002), a $[\mathrm{OH}] /\left[\mathrm{H}_{2} \mathrm{O}\right]$ abundance ratio of $0.1-1$ is estimated for the hot layer, which, according to the authors, points towards the presence of a strong UV field illuminating the outer shells of the cloud. The high abundance of $\mathrm{H}_{2} \mathrm{O}$ estimated by us does not allow to discriminate among the proposed mechanisms, and it is anyway likely that all of them contribute, to some extent, to the anomalous heating of this region. However, our radiative transfer calculations allow us to separate the chemistry driving the water abundance in the hot layer from that taking place in the warm envelope, thus helping to set more solid constraints on the physical characteristics of the hot diffuse gas, such as, for example, its spatial extent.

We would also like to stress the high deuterium fractionation $\left([\mathrm{HDO}] /\left[\mathrm{H}_{2} \mathrm{O}\right] \sim 6.4 \times 10^{-4}\right)$ inferred, for the SgrB2 hot cores, by our radiative transfer model. Our value is in agreement (about a factor of 3 higher) with that estimated by Gensheimer et al. (1996) for SgrB2(N), and it is almost 400 times larger than the elemental $[\mathrm{D}] /[\mathrm{H}]$ 
Table 7. Description of the radial profiles of density and temperature used to model the hot cores and warm envelope (HC and WE respectively, Sect. 4.2), and the hot layer (HL, see Sect. 4.3) of the SgrB2 cloud complex. The profiles are described by the coefficients $a_{0}, a_{1}, r_{0}$ and $\alpha$ according to the law: $P(r)=a_{0}+a_{1} \cdot\left(r / r_{0}\right)^{\alpha}$. The radii are (cf. Fig. 4): $r<0.05 \mathrm{pc}$ for the hot core and $0.05 \mathrm{pc} \leq r \leq 22.5 \mathrm{pc}$ for the warm envelope. The hot layer is, in our model, $0.02 \mathrm{pc}$ thick.

\begin{tabular}{|c|c|c|c|c|c|c|c|c|c|c|c|c|}
\hline \multirow[b]{2}{*}{ Region } & \multicolumn{4}{|c|}{$\bar{n} n\left(\mathrm{H}_{2}\right)$} & \multicolumn{4}{|c|}{$\overline{\overline{T_{\text {gas }}}}$} & \multicolumn{4}{|c|}{$\bar{T} T_{\text {dust }}$} \\
\hline & $\begin{array}{c}a_{0} \\
\left(\mathrm{~cm}^{-3}\right)\end{array}$ & $\begin{array}{c}a_{1} \\
\left(\mathrm{~cm}^{-3}\right)\end{array}$ & $\begin{array}{c}r_{0} \\
(\mathrm{pc})\end{array}$ & $\alpha$ & $\begin{array}{c}a_{0} \\
(\mathrm{~K})\end{array}$ & $\begin{array}{c}a_{1} \\
(\mathrm{~K})\end{array}$ & $\begin{array}{c}r_{0} \\
(\mathrm{pc})\end{array}$ & $\alpha$ & $\begin{array}{c}a_{0} \\
(\mathrm{~K})\end{array}$ & $\begin{array}{c}a_{1} \\
(\mathrm{~K})\end{array}$ & $\begin{array}{c}r_{0} \\
(\mathrm{pc})\end{array}$ & $\alpha$ \\
\hline $\mathrm{HC}$ & $3.4 \times 10^{7}$ & 0 & - & - & 200 & 0 & - & - & 200 & 0 & - & - \\
\hline WE & $2.2 \times 10^{3}$ & $5.5 \times 10^{4}$ & 1.25 & -2 & 0 & 40 & 1 & -0.5 & 0 & 40 & 1 & -0.5 \\
\hline HL & $10^{3}$ & 0 & - & - & 500 & 0 & - & - & - & - & - & - \\
\hline
\end{tabular}

Table 8. Summary of the peak column densities of $\mathrm{H}_{2}, \mathrm{HDO}$ and $\mathrm{H}_{2} \mathrm{O}$ derived in Sects. 4.2 and 4.3. The values relative to hot cores and warm envelope $(\mathrm{HC}+\mathrm{WE})$ are based on the radiative transfer code illustrated in $\mathrm{Z95b}$ and modified as in Sect. 4.1. The determination of the hot layer values is described in Sect. 4.3. The $[\mathrm{HDO}] /\left[\mathrm{H}_{2} \mathrm{O}\right]$ ratio for each region is also indicated. The value of $[\mathrm{HDO}] /\left[\mathrm{H}_{2} \mathrm{O}\right]$ in the hot layer is assumed equal to the $[\mathrm{D}] /[\mathrm{H}]$ ratio in the Galactic Center (cf. Lubowich et al. 2000).

\begin{tabular}{ccccc}
\hline \hline & $\begin{array}{c}N\left(\mathrm{H}_{2}\right) \\
\left(\mathrm{cm}^{-2}\right)\end{array}$ & $\begin{array}{c}N(\mathrm{HDO}) \\
\left(\mathrm{cm}^{-2}\right)\end{array}$ & $\begin{array}{c}N\left(\mathrm{H}_{2} \mathrm{O}\right) \\
\left(\mathrm{cm}^{-2}\right)\end{array}$ & {$[\mathrm{HDO}] /\left[\mathrm{H}_{2} \mathrm{O}\right]$} \\
Region & $1.9 \times 10^{25}$ & $2.3 \times 10^{16}$ & $3.6 \times 10^{19}$ & $6.4 \times 10^{-4}$ \\
HC+WE & $6 \times 10^{19}$ & $5.2 \times 10^{10}$ & $3.5 \times 10^{16}$ & $1.7 \times 10^{-6(\star)}$ \\
HL & & & &
\end{tabular}

(^) Assumed (cf. Lubowich et al. 2000).

ratio measured towards the Galactic Center $^{6}$ (Lubowich et al. 2000). An enhancement of the deuterium fractionation in hotcore-type sources has been observed for a variety of chemical species $\left(\left[\mathrm{CH}_{3} \mathrm{OD}\right] /\left[\mathrm{CH}_{3} \mathrm{OH}\right]\right.$, Mauersberger et al. 1988; $\left[\mathrm{NH}_{2} \mathrm{D}\right] /\left[\mathrm{NH}_{3}\right]$, Jacq et al. 1990; [DCN]/[HCN], Schilke et al. 1992, Hatchell et al. 1998; [DCO $\left.{ }^{+}\right] /\left[\mathrm{HCO}^{+}\right]$, Jacq et al. 1999; $[\mathrm{HDS}] /\left[\mathrm{H}_{2} \mathrm{~S}\right]$, Hatchell et al.1999; $\left[\mathrm{CH}_{2} \mathrm{DCN}\right] /\left[\mathrm{CH}_{3} \mathrm{CN}\right]$, Gerin et al. 1992; and [HDO]/[H $\left.\mathrm{H}_{2} \mathrm{O}\right]$, Jacq et al. 1990, 1999; Gensheimer et al. 1996; Pardo et al. 2001), and is generally attributed to evaporation of deuterated species from grain mantles due to the formation of an embedded heating source (see Walmsley et al. 1987). It must be kept in mind that the quoted estimates of the $[\mathrm{D}] /[\mathrm{H}]$ ratio in the Galactic Center are affected by large uncertainties (about 1 order of magnitude). However, we can assume our result to be in agreement with the general finding that the abundance ratio of deuterated species to their non-deuterated counterparts is, in hot-core-type sources (cf. references above), enhanced by a factor of a few $10^{2}$ with respect to the elemental $[\mathrm{D}] /[\mathrm{H}]$ ratio. Thus, the value of $[\mathrm{HDO}] /\left[\mathrm{H}_{2} \mathrm{O}\right]$ derived in Sect. 4.2 yields a $[\mathrm{D}] /[\mathrm{H}]$ ratio of a few $10^{-6}$, supporting the hypothesis of a lower $[\mathrm{D}] /[\mathrm{H}]$ ratio in The Galactic Center region.

Acknowledgements. The authors are grateful to D. Neufeld for providing the 548-GHz SWAS data in digital format; to M. Walmsley, T. Wilson and C. Ceccarelli for their valuable comments; and to the referee, E. Bergin, whose input has very much contributed to improve the quality of this paper. CC acknowledges travel support and sunny hospitality from the Submillimeter Wave Astrophysics Research Group at Caltech.

\footnotetext{
${ }^{6}$ The ratio of the abundance of a deuterated species with respect to its non-deuterated counterpart, $[\mathrm{XD}] /[\mathrm{XH}]$, is expected to scale with the $[\mathrm{D}] /[\mathrm{H}]$ ratio in the gas (Gerin \& Roueff 1999; E. Bergin, priv. comm.).
}

\section{References}

Bergin, E. A., Melnick, G. J., \& Neufeld, D. A. 1998, ApJ, 499, 777 Bergin, E. A., Neufeld, D. A., \& Melnick, G. J. 1999, ApJ, 510, L145 Ceccarelli, C., Hollenbach, D. J., \& Tielens, A. G. G. M. 1996, ApJ, 471,400

Ceccarelli, C., Baluteau, J.-P., Walmsley, C. M., et al. 2002, A\&A, $383,603(\mathrm{C} 02)$

Cernicharo, J., Lim, T., Cox, P., et al. 1997, A\&A, 323, 25

Dowell, C. D., Lis, D. C., Serabyn, E., et al. 1999, The Central Parsecs of the Galaxy, ASP Conf. Ser., 186, 453

Elitzur, M., \& de Jong, T., A\&A, 67, 323

Flower, D. R., \& Pineau des Forêts, G. 1994, MNRAS, 268, 724

Flower, D. R., Pineau des Forêts, G., \& Walmsley, C. M. 1995, A\&A, 294, 815

Gensheimer, P. D., Mauersberger, R., \& Wilson, T. L. 1996, A\&A, 314,281

Gerin, M., Combes, F., Wlodarczak, G., et al. 1992, A\&A, 259, 35

Gerin, M., \& Roueff, E. 1999, Highly Redshifted Radio Lines, ASP Conf. Ser., 156, 196

Goicoechea, J. R., Cernicharo, J., \& Caux, E. 2000, ISO beyond the peaks: The 2nd ISO workshop on analytical spectroscopy, ed. A. Salama, M. F. Kessler, K. Leech, \& B. Schulz, ESA-SP 456, 99

Goicoechea, J. R., \& Cernicharo, J. 2001, ApJ, 554, 213

Goicoechea, J. R., \& Cernicharo, J. 2002, ApJ, 576, L77

Goldsmith, P. F., Snell, R. L., \& Lis, D. C. 1987, ApJ, 313, 5

Goldsmith, P. F., Lis, D. C., Hills, R., \& Lasenby, J. 1990, ApJ, 350, 186

Goldsmith, P. F., Lis, D. C., Lester, D. F., \& Harvey, P. M. 1992, ApJ, 389,338

Gordon, M. A., Berkermann, U., Mezger, P. G., et al. 1993, A\&A, 280, 208

Greaves, J. S., White, G. J., Ohishi, M., Hasegawa, T., \& Sunada, K. 1992, A\&A, 260, 381

Hasegawa, T., Sato, F., Whiteoak, J. B., \& Miyawaki, R. 1994, ApJ, 429, L77 
Hatchell, J., Millar, T. J., \& Rodgers, S. D. 1998, A\&A, 332, 695

Hatchell, J., Roberts, H., \& Millar, T. J. 1999, A\&A, 346, 227

Helmich, F. P., van Dishoeck, E. F., \& Jansen, D. J. 1996, A\&A, 313, 657

Henkel, C., Mauersberger, R., Wilson, T. L., et al. 1987, A\&A, 182, 299

Hüttemeister, S., Wilson, T. L., Henkel, C., \& Mauersberger, R. 1993, A\&A, 276, 445

Hüttemeister, S., Wilson, T. L., Mauersberger, R., et al. 1995, A\&A, 294, 667

Jacq, T., Jewell, P. R., Henkel, C., Walmsley, C. M., \& Baudry, A. 1988, A\&A, 199, 5

Jacq, T., Walmsley, C. M., Henkel, C., et al. 1990, A\&A, 228, 447

Jacq, T., Baudry, A., Walmsley, C. M., \& Caselli, P. 1999, A\&A, 347, 957

Kooi, J., Kawamura, J., Chen, J., et al. 2000, Int J. IR and MM Waves, 21,9

Linsky, J. L. 1998, Space Sci. Rev., 84, 285

Lis, D. C., \& Goldsmith, P. F. 1989, ApJ, 337, 704 (LG89)

Lis, D. C., Goldsmith, P. F., Carlstrom, J. E., \& Scoville, N. Z. 1993, ApJ, 402, 238 (L93)

Lis, D. C., Keene, J., Phillips, T. G., et al. 2001, ApJ, 561, 823

Lubowich, D. A., Pasachoff, J. M., Balonek, T. J., et al. 2000, Lett. Nat., 405, 1025

Martín-Pintado, J., de Vicente, P., Wilson, T. L., \& Johnston, K. J. 1990, A\&A, 236, 193

Martín-Pintado, J., de Vicente, P., Rodríguez-Fernández, N. J., Fuente, A., \& Panesas, P. 2000, A\&A, 356, L5

Mauersberger, R., Henkel, C., Jacq, T., \& Walmsley, C. M. 1988, A\&A, 194, 1

Melnick, G. J., Ashby, M. L. N., Plume, R., et al. 2000, ApJ, 539, 87

Meyer, D. M., Jura, M., \& Cardelli, J. A. 1998, ApJ, 493, 222

Miao, Y., \& Snyder, L. E. 1997, ApJ, 480, L67

Neufeld, D. A., \& Melnick, G. J. 1987, ApJ, 322, 266

Neufeld, D. A., Zmuidzinas, J., Schilke, P., \& Phillips, T. G. 1997, ApJ, 488, 141

Neufeld, D. A., Ashby, M. L. N., Bergin, E. A., et al. 2000, ApJ, 539, 111

Neufeld, D. A., Bergin, E. A., Goldsmith, P. F., \& Melnick, G. J. 2003, in preparation

Nummelin, A., Bergman, P., Hjalmarson, Å., et al. 2000, ApJS, 128, 213

Pardo, J. R., Cernicharo, J., Herpin, F., et al. 2001, ApJ, 562, 799
Phillips, T. G., Scoville, N. Z., Kwan, J., Huggins, P. J., \& Wannier, P. G. 1978, ApJ, 222, 59

Polehampton, E. T., Baluteau, J.-P., Ceccarelli, C., Swinyard, B. M., \& Caux, E. 2002, A\&A, 388, L44

Roberts, H., \& Millar, T. J. 2000, A\&A, 364, 780

Rodgers, S. D., \& Millar, T. J. 1996, MNRAS, 280, 1046

Rolleston, W. R. J., Smartt, S. J., Dufton, P. L., \& Ryans, R. S. I. 2000, A\&A, 363, 537

Schilke, P., Walmsley, C. M., Pineau des Forêts, G., et al. 1992, A\&A, 256, 595

Schulz, A., Güsten, R., Serabyn, E., \& Walmsley, C. M. 1991, A\&A, 246, 55

Shaver, P. A., McGee, R. X., Newton, L. M., Danks, A. C., \& Pottasch, S. R. 1983, MNRAS, 204, 53

Snell, R. L., Howe, J. E., Ashby, M. L. N., et al. 2000a, ApJ, 539, 93

Snell, R. L., Howe, J. E., Ashby, M. L. N., et al. 2000b, ApJ, 539, 101

Snyder, L. E., Kuan, Y.-J., \& Miao, Y. 1994, The Structure and Content of Molecular Clouds, ed. T. L. Wilson, \& K. J. Johnston (Berlin: Springer), 187

Tieftrunk, A., Pineau des Forêts, G., Schilke, P., \& Walmsley, C. M. 1994, A\&A, 289, 579

Timmermann, R., Poglitsch, A., \& Nikola, T. 1996, ApJ, 460, 65

van Dishoeck, E. F., Jansen, D. J., Schilke, P., \& Phillips, T. G. 1993, ApJ, 416, 83

van Dishoeck, E. F., \& Helmich, F. P. 1996, A\&A, 315, 177

Vogel, S. N., Genzel, R., \& Palmer, P. 1987, ApJ, 316, 243

Walmsley, C. M., Hermsen, W., Henkel, C., Mauersberger, R., \& Wilson, T. L. 1987, A\&A, 172, 311

Walmsley, C. M., Bachiller, R., Pineau des Forêts, G., \& Schilke, P. 2002, ApJ, 566, 109

Whiteoak, J. B., \& Gardner, F. F. 1979, MNRAS, 188, 445

Whiteoak, J. B., \& Gardner, F. F. 1981, MNRAS, 197, 39

Williams, D. A. 1993, in Dust and Chemistry in Astronomy, T. J. Millar, \& D. A. Williams (Philadelphia: IPP Bristol)

Wilson, T. L., Ruf, K., Walmsley, C. M., et al. 1982, A\&A, 115, 185

Wright, C. M., van Dishoeck, E. F., Black, J. H., et al. 2000, A\&A, 358, 689

Zmuidzinas, J., Blake, G. A., Carlstrom, J., et al. 1995b, Proc. of the Airborne Astronomy Symp. on the Galactic Ecosystem: From Gas to Stars to Dust, ed. M. R. Haas, J. A. Davidson, \& E. F. Erickson (San Francisco: ASP) (Z95a)

Zmuidzinas, J., Blake, G. A., Carlstrom, J., Keene, J., \& Miller, D. 1995, ApJ, 447, L125 (Z95b) 